\title{
INAPPROXIMABILITY OF THE KIDNEY EXCHANGE PROBLEM ${ }^{*}$
}

\author{
Péter Biró ${ }^{1}$ and Katarína Cechlárová ${ }^{2}$ \\ ${ }^{1}$ Department of Algebra and Department of Computer Science and Information Theory, \\ Budapest University of Technology and Economics, Hungary \\ email: pbiro@cs.bme.hu \\ ${ }^{2}$ Institute of Mathematics, P.J. Šafárik University, Jesenná 5, 04154 Košice, Slovakia \\ email: katarina.cechlarova@upjs.sk
}

June 22, 2006

\begin{abstract}
To overcome the shortage of kidneys available for transplantation, several countries have started various programmes of kidney exchanges between incompatible patient-donor pairs. This situation can be modelled as a cooperative game in which the players are the patient-donor pairs and their preferences are derived in the first step from the suitability of the donated kidney and in the second step from the length of the obtained cycle of exchanges. Although the core of such a cooperative game is always nonempty and one core solution can be found by the famous Top Trading Cycles algorithm, many questions concerning the structure of the core are NP-hard. In this paper we show that the problem of finding a core permutation that maximizes the number of patients who obtain a suitable kidney is not approximable within $n^{1-\varepsilon}$ for any $\varepsilon>0$ unless $P=N P$.
\end{abstract}

Keywords. Kidney transplantation, cooperative game, core, inapproximability.

\section{Introduction}

The most effective treatment for kidney failure that is currently known is transplantation. However, the number of patients grows very fast and the supply of cadaveric kidneys is insufficient (for the most recent statistical data see e.g. [16]). Moreover, very often the kidney from a willing living donor, genetic or emotional relative of a patient, is not suitable for immunological reasons.

*This work was supported by the VEGA grant 1/3001/06, Science and Technology Assistance Agency contract No. APVT-20-004104 
Therefore in several countries various kidney exchange programmes have been established to systematically search for incompatible patient-donor pairs and possible kidney exchanges between them: in Romania [9], the Netherlands [8], USA [13]; other countries have at least changed their legislation to enable such exchanges [6]. All practical experiences as well as simulation studies (for example $[12,13,14])$ show that kidney exchanges really help to increase the number of transplantations.

Most transplantation centres organize just exchanges between two pairs [8, $11,14]$, however, allowing longer cycles might lead to better results $[12,13]$. Moreover, it is also important to try to balance the desire of an individual patient for the best possible outcome for him with the need for a socially optimal solution and this is just the place for an application of game theory models and methods. Therefore we follow the approach started in [10] and developed in [3], in that we represent kidney exchange as a cooperative game in which patient-donor pairs seek cyclic exchanges of kidneys. Preferences of players take into account not only the suitability of the received kidney, but also the length of the obtained cycle, as it is an accepted rule that all operations on a cycle should be performed simultaneously [13] and with longer cycles logistics becomes more complicated.

In this paper we concentrate on the core of the considered kidney exchange game. With no indifferences, it is always nonempty and the famous Top Trading Cycles algorithm, originally proposed by Gale in [15] for housing markets, can be used to find a core permutation for each kidney exchange game instance [2]. On the other hand, in [1] it was proved that in the case with indifferences it is NP-complete to decide whether the core is nonempty. However, the case with strict preferences is also not so simple. The core may contain many pemutations and an efficient description of its structure is unlikely to exist, as in [4] and [7] several decision problems concerning the structure of the core were proved to be NP-complete.

In this paper we concentrate on the problem of maximizing the number of players covered by a core permutation, as this is the number of patients for whom a suitable donor has been found. We give a very pessimistic result showing that unless $P=N P$, this problem is not approximable within $n^{1-\varepsilon}$ for any $\varepsilon>0$.

\section{The Kidney Exchange game}

An instance of the kidney exchange game is represented by a directed graph $G=(V, A)$ without loops where each vertex $i \in V$ corresponds to a patient and his intended incompatible donor (or donors). A pair $(i, j) \in A$ if the patient associated to vertex $i$ can accept a kidney from a donor associated to vertex $j$. Moreover, for each vertex $i$ there is a linear ordering $\preceq_{i}$ on the set of endvertices of arcs incident from $i$, where $j \preceq_{i} k$ means that vertex $j$ is at least as good for vertex $i$ as vertex $k$. This ordering is represented by a preference list $P(i)$ of $i$. 
To keep things simple, we shall usually not give the graph $G$ explicitly, as its structure is implied by the contents of the preference lists.

In this paper we suppose that preference lists do not contain ties, i.e. if $k \preceq_{i} j$ then $j \preceq_{i} k$ does not hold; we also write $k \prec_{i} j$ in this case and $k \sim_{i} j$ otherwise.

For brevity, the first entry in $P(i)$ will usually be called the favourite of $i$ and denoted by $f(i)$.

Definition 1 An instance of the kidney exchange game (KE game for short) is given by a triple $\Gamma=(V, G, \mathcal{O})$, where $V$ is the set of players, $G$ is a digraph with the vertex set $V$ and $\mathcal{O}=\left\{\preceq_{i} ; i \in V\right\}$. A solution of $\Gamma$ is a permutation $\pi$ of $V$ such that for each $i \in V$, the inequality $i \neq \pi(i)$ implies $(i, \pi(i)) \in A$.

A player $i$ evaluates a permutation $\pi$ not only according to the player (kidney) $\pi(i)$ he is assigned to (and called the successor of $i$ in $\pi$ ), but he also takes into account the length of the cycle he is contained in and hence he considers pair $\left(\pi(i), C^{\pi}(i)\right)$, where $C^{\pi}(i)$ denotes the cycle of $\pi$ containing $i$. If $C^{\pi}(i)$ has length at least 2 , then player $i$ is said to be covered, otherwise he is uncovered by $\pi$. The symbol $\operatorname{cov}(\pi)$ denotes the number of players covered by permutation $\pi$.

Preferences from $\mathcal{O}$ are extended to preferences over (player,cycle) pairs by the following definition. (The same symbol is used for preferences over players as well as over (player,cycle) pairs, as no confusion should arise.)

Definition 2 A player $i$ prefers pair $(j, M)$ to pair $(k, N)$ if

(i) $j \prec_{i} k$ or

(ii) $j \sim_{i} k$ and $|M|<|N|$.

Definition $3 A$ coalition $S \subseteq V$ blocks a solution $\pi$ if there exists a permutation $\sigma$ of $S$ such that each player $i \in S$ prefers $\left(\sigma(i), C^{\sigma}(i)\right)$ to $\left(\pi(i), C^{\pi}(i)\right)$. A permutation $\pi$ is in the core $\operatorname{Core}(\Gamma)$ of a KE game instance $\Gamma$ if no coalition blocks $\pi$.

For brevity, we shall often refer to a blocking set $S$ considered together with the improving permutation $\sigma$ as a blocking cycle. Further, let us denote

$$
\operatorname{cov}(\Gamma)=\max \{\operatorname{cov}(\pi), \pi \in \operatorname{Core}(\Gamma)\}
$$

In [4] the problem of deciding whether $\operatorname{cov}(\Gamma)=|V|$ was proved to be NPcomplete. In this paper we study the problem MAX-COVER-KE, i.e. the problem of finding, given a KE game instance $\Gamma$, a core permutation $\pi$ such that $\operatorname{cov}(\Gamma)=$ $\operatorname{cov}(\pi)$. We show that MAX-COVER-KE is not approximable within $n^{1-\varepsilon}$ for any $\varepsilon>0$, unless $P=N P$. In our inapproximability proof we shall use the problem CUBIC-MIN-MM, which is the problem of deciding, given a cubic graph $G$ and an integer $K$, whether $G$ admits a maximal matching of size not exceeding $K$. CUBIC-MIN-MM was proved to be NP-complete in [5]. 


\section{Inapproximability of the maximum number of exchanges}

Theorem 1 MAX-COVER-KE is not approximable within $n^{1-\varepsilon}$ for any $\varepsilon>0$ unless $P=N P$.

Proof. Let $\varepsilon>0$ be given. Let a cubic graph $G=(V, E)$ and a positive integer $K$ as an instance $I$ of CUBIC-MIN-MM be given. Let $V=\left\{v_{1}, v_{2}, \ldots, v_{p}\right\}$ and suppose that the three vertices adjacent to vertex $v_{i}, i=1,2, \ldots, p$, are $v_{i_{1}}, v_{i_{2}}, v_{i_{3}}$ where we always suppose that their indices are ordered $i_{1}<i_{2}<i_{3}$. Further denote

$$
K^{\prime}=p-2 K, s=2 p+3 K^{\prime}=5 p-6 K, t=\left\lceil\frac{1}{\varepsilon}\right\rceil, N=(10 p)^{t} .
$$

Notice that $t>1, t \varepsilon \geq 1$.

We create an instance $\Gamma=\left(V^{\prime}, E^{\prime}, \mathcal{O}\right)$ of the $\mathrm{KE}$ game, in which the set of players will be $U \cup U^{\prime} \cup H \cup X$, where $U=\left\{u_{1}, \ldots, u_{p}\right\}, U^{\prime}=\left\{u_{1}^{\prime}, \ldots, u_{p}^{\prime}\right\}$, $H=\left\{h_{k}^{j}, k=1,2, \ldots, K^{\prime}, j=1,2,3\right\}, X=\{x, y, z\} \cup\left\{c_{1}, \ldots, c_{N-3}\right\}$. Notice that $s=\left|U \cup U^{\prime} \cup H\right|$ and the number of players in $\Gamma$ is $n=s+(10 p)^{t}$, which is polynomial in size of $I$.

Preferences of players are given in Figure 1.

\begin{tabular}{lr}
\hline$\left(u_{i}\right): u_{i}^{\prime}$ & for $i=1,2, \ldots, p$ \\
$P\left(u_{i}^{\prime}\right): u_{i_{1}}, u_{i_{2}}, u_{i_{3}}, h_{1}^{1}, h_{2}^{1}, \ldots, h_{K^{\prime}}^{1}$ & for $i=1,2, \ldots, p$ \\
$P\left(h_{k}^{1}\right): h_{k}^{2}$ & for $k=1,2, \ldots, K^{\prime}$ \\
$P\left(h_{k}^{2}\right): h_{k}^{3}$ & for $k=1,2, \ldots, K^{\prime}$ \\
$P\left(h_{k}^{3}\right): u_{1}, u_{2}, \ldots, u_{p}, x$ & for $k=1,2, \ldots, K^{\prime}$ \\
$P(x): y$ & \\
$P(y): z$ & \\
$P(z): h_{1}^{1}, h_{2}^{1}, \ldots, h_{K^{\prime}}^{1}, c_{1}$ & \\
$P\left(c_{j}\right): c_{j+1}$ & \\
$P\left(c_{N-3}\right): x$ &
\end{tabular}

Figure 1: Preferences of players

The constructed graph $G^{\prime}$ contains no cycles of the length less than 4 , however, there are 4-cycles of the form $\left(u_{i}, u_{i}^{\prime}, u_{j}, u_{j}^{\prime}\right)$ for $\left\{v_{i}, v_{j}\right\} \in E, 5$-cycles of the form $\left(u_{i}, u_{i}^{\prime}, h_{k}^{1}, h_{k}^{2}, h_{k}^{3}\right)$ for each $i=1, \ldots, p, k=1, \ldots, K^{\prime}$ and 6 -cycles 
$\left(h_{k}^{1}, h_{k}^{2}, h_{k}^{3}, x, y, z\right)$ for each $k=1, \ldots, K^{\prime}$. Moreover, there is one long cycle $C_{N}=\left(x, y, z, c_{1}, \ldots, c_{N-3}\right)$ of length $N$. Every other cycle in $G^{\prime}$ contains a set of vertices that form one of the listed cycles.

Now suppose that $G$ contains a maximal matching $M$ with $|M| \leq K$, say $|M|=L$. Let $L^{\prime}=p-2 L$. Let $X=\left\{v_{r_{1}}, v_{r_{2}}, \ldots, v_{r_{L^{\prime}}}\right\} \subset V$ be the set of vertices of $G$ not covered by $M$, suppose that $r_{1}<r_{2}<\ldots<r_{L^{\prime}}$. Notice that $L \leq K$ implies $L^{\prime} \geq K^{\prime}$.

We construct a permutation $\pi \in \operatorname{Core}(\Gamma)$ such that $\operatorname{cov}(\pi) \geq(10 p)^{t}$.

First, for each $\left\{v_{i}, v_{j}\right\} \in M$ we let $\left(u_{i}, u_{i}^{\prime}, u_{j}, u_{j}^{\prime}\right) \in \pi$.

Now add to $\pi$ cycles $\left(u_{r_{i}}, u_{r_{i}}^{\prime}, h_{i}^{1}, h_{i}^{2}, h_{i}^{3}\right)$ for $i=1,2, \ldots, K^{\prime}$. Notice that all players in $H$ are covered while some players in $U \cup U^{\prime}$ may remain uncovered, but then their indices are greater than $r_{K^{\prime}}$. Finally, $C_{N}=\left(x, y, z, c_{1}, \ldots, c_{N-3}\right) \in \pi$.

To show that $\pi \in \operatorname{Core}(\Gamma)$, let us consider all the players in turn.

Players $u_{i}$ from 4 -cycles of $\pi$ cannot improve as $\pi\left(u_{i}\right)=f\left(u_{i}\right)$ and $C^{\pi}\left(u_{i}\right)$ is shortest possible. A player $u_{i}$ lying on a 5 -cycle of $\pi$ could improve by getting a shorter cycle, but as $M$ is maximal, such a cycle would necessarily contain some player $u_{j}$ who already is on a 4 -cycle and hence cannot improve. An uncovered player $u_{i}$ prefers to be on a 4-cycle (which is impossible as above) or to be on a 5 -cycle containing a player $h_{j}^{3}$, but $\pi\left(h_{j}^{3}\right)$ for each $j$ is equal to some player $u_{k}$ with $k<i$, hence $h_{j}^{3}$ would not improve by joining $u_{i}$.

As a result, no player $u_{i}^{\prime}, i=1,2, \ldots, p$ can improve, as each cycle containing $u_{i}^{\prime}$ also contains $u_{i}$. Further, each player from $H$ is on a 5 -cycle and can neither get a shorter cycle nor a better successor than the one he has in $\pi$. Finally, players $x, y, z$ could improve on a 6 -cycle containing players from $H$, but the previous sentence implies that these will not enable such a blocking.

So $\pi \in \operatorname{Core}(\Gamma)$ and $\operatorname{cov}(\pi) \geq(10 p)^{t}$.

Conversely, we shall show that if $G$ does not have a maximal matching of size not exceeding $K$, then $\operatorname{cov}(\pi) \leq s+3$ for each permutation $\pi \in \operatorname{Core}(\Gamma)$.

To get a contradiction, suppose that there exists $\pi \in \operatorname{Core}(\Gamma)$ with $\operatorname{cov}(\pi)>$ $s+3$. Then $\pi$ must contain the long cycle $C_{N}$.

First we will prove that $|C| \leq 5$ for each cycle $C \in \pi, C \neq C_{N}$. Therefore suppose that $|C| \geq 6$ for some $C \in \pi, C \neq C_{N}$. Then $C$ contains an arc $\left(u_{i}, u_{i}^{\prime}\right)$ for some $i \in\{1,2, \ldots, p\}$, let $i$ be the smallest index of $\left(u_{i}, u_{i}^{\prime}\right) \in C$. If $C=\left(u_{i}, u_{i}^{\prime}, h_{k}^{1}, h_{k}^{2}, h_{k}^{3}, u_{j}, \ldots\right)$, then the cycle $Z=\left(u_{i}, u_{i}^{\prime}, h_{k}^{1}, h_{k}^{2}, h_{k}^{3}\right)$ will be blocking, since all players of $Z$ except for $h_{k}^{3}$ have the same successor and a shorter cycle and $h_{k}^{3}$ has a better successor, as he prefers $u_{i}$ to $u_{j}$. If $C=\left(u_{i}, u_{i}^{\prime}, u_{j}, u_{j}^{\prime}, \ldots\right)$ then $Z=\left(u_{i}, u_{i}^{\prime}, u_{j}, u_{j}^{\prime}\right)$ blocks as all the players of $Z$ except for $u_{j}^{\prime}$ have the same successor and a shorter cycle and $u_{j}^{\prime}$ has a better successor.

Now create a matching $M$ of $G$ by setting $\left\{v_{i}, v_{j}\right\} \in M$ iff the 4-cycle $\left(u_{i}, u_{i}^{\prime}, u_{j}, u_{j}^{\prime}\right)$ belongs to $\pi$. $M$ must be a matching as cycles of a permutation are disjoint. To show that $M$ is maximal, suppose that edge $\left\{u_{k}, u_{\ell}\right\}$ can be added to $M$. Then players $u_{k}, u_{k}^{\prime}, u_{\ell}, u_{\ell}^{\prime}$ are all either on 5-cycles or uncovered, but then the cycle $\left(u_{k}, u_{k}^{\prime}, u_{\ell}, u_{\ell}^{\prime}\right)$ will be blocking. Further, since $C_{N} \in \pi$, 
each player of $H$ must be covered so as not to allow a blocking cycle of the form $\left(x, y, z, h_{j}^{1}, h_{j}^{2}, h_{j}^{3}\right)$. This means that $K^{\prime}$ players of $U$ are in 5-cycles, leaving $p-K^{\prime}=p-(p-2 K)=2 K$ players from $U$ available for the definition of $M$. Hence $|M| \leq K$, a contradiction.

The presented reduction shows that unless $P=N P$, it is impossible to distinguish between instances $\Gamma$ of the KE game with $\operatorname{cov}(\Gamma) \leq s+3$ and those with $\operatorname{cov}(\Gamma) \geq(10 p)^{t}$. Now we prove the stated inapproximability result.

Suppose that there exists a polynomial algorithm $\mathcal{A}$ such that its output $\mathcal{A}(\Gamma) \geq \frac{\operatorname{cov}(\Gamma)}{n^{1-\varepsilon}}$ for each instance $\Gamma$ of the KE game. If this algorithm gets for the input an instance $\Gamma$ of the form constructed which has $\operatorname{cov}(\Gamma) \geq(10 p)^{t}$, it must obtain

$$
\begin{aligned}
\mathcal{A}(\Gamma) & \geq \frac{(10 p)^{t}}{n^{1-\varepsilon}}=\frac{(10 p)^{t}}{\left(5 p-6 K+(10 p)^{t}\right)^{1-\varepsilon}} \\
& \geq \frac{(10 p)^{t}}{\left(5 p+(10 p)^{t}\right)^{1-\varepsilon}} \quad \text { as } 1-\varepsilon>0 \text { and } 5 p-6 K+(10 p)^{t}<5 p+(10 p)^{t} \\
& =\frac{\left(5 p+(10 p)^{t}\right)^{\varepsilon}}{\frac{5 p+(10 p)^{t}}{(10 p)^{t}}}=\frac{\left(5 p+(10 p)^{t}\right)^{\varepsilon}}{\frac{5 p}{(10 p)^{t}}+1} \\
& \geq \frac{\left(5 p+(10 p)^{t}\right)^{\varepsilon}}{2} \geq \frac{\left((10 p)^{t}\right)^{\varepsilon}}{2} \\
& \geq \frac{10 p}{2}=5 p>5 p-6 K+3=s+3
\end{aligned}
$$

so such an algorithm will be able to decide CUBIC-MIN-MM in polynomial time, a contradiction.

\section{Acknowledgement}

The authors would like to thank David Manlove for discussions and useful suggestions.

\section{References}

[1] K. Cechlárová and J. Hajduková, Computational complexity of stable partitions with $\mathcal{B}$-preferences, Int. J. Game Theory 31 (2002) 3, 353-364.

[2] K. Cechlárová and A. Romero-Medina, Stability in coalition formation games, International Journal of Game Theory 29, 2001, 487-494.

[3] K. Cechlárová, T. Fleiner and D. Manlove, The kidney exchange game, Proc. SOR '05 (2005), Eds. L. Zadnik-Stirn, S. Drobne, 77-83. 
[4] K. Cechlárová and V. Lacko, The kidney exchange problem: How hard is it to find a donor?, IM Preprint 4/2006 (2006).

[5] J.D. Horton and K. Kilakos, Minimum edge dominating sets, SIAM J. Discrete Mathematics, 375-387 (1993).

[6] Human Tissue Authority, London, http://www.hta.gov.uk/

[7] R. W. Irving, The Cycle Stable Roommates, personal communication (2006).

[8] K.M. Keizer, M. de Klerk, B.J.J.M. Haase-Kromwijk, W. Weimar, The Dutch algorithm for allocation in living donor kidney exchange, Transplantation Proceedings 37, 589-591 (2005).

[9] M. Lucan, P. Rotariu, D. Neculoiu and G. Iacob, Kidney exchange program: a viable alternative in countries with low rate of cadaver harvesting, Transplantation Proceedings 35, 933-934 (2003).

[10] A. Roth, T. Sönmez and U. Ünver, Kidney Exchange, Quarterly J. of Econ. 119: 457-488 (2004).

[11] A. Roth, T. Sönmez and U. Ünver, Pairwise Kidney Exchange, J. Econ. Theory, Journal of Economic Theory 125/2, 151-188 (2005).

[12] A. Roth, T. Sönmez and U. Ünver, Efficient Kidney Exchange: Coincidence of Wants in a Structured Market, manuscript, (NBER Paper w11402) (2005).

[13] S.L. Saidman, A. Roth, T. Sönmez, U. Ünver, and F. L. Delmonico, Increasing the Opportunity of Live Kidney Donation By Matching for Two and Three Way Exchanges, Transplantation 81/5, 773-782 (2006).

[14] D.L. Segev, S.E. Gentry, D.S. Warren, B. Reeb, R.A. Montgomery, Kidney Paired donation and optimizing the use of live donor organs, JAMA 293/15, 1883-1890 (2005).

[15] L. Shapley and H. Scarf, On cores and indivisibility, J. Math. Econ. 1:23-37 (1974).

[16] United Network for Organ Sharing, http://www.optn.org 